\title{
Antioxidants retard the ageing of mouse oocytes
}

\author{
YEXING XIAN $^{1}$, LIFENG LIANG ${ }^{1}$, SHUTAO QI $^{1}$, YINGJUN XIE $^{1}$, BING SONG $^{1}$, \\ SHUMING OUYANG ${ }^{1}$, YUHUAN XIE ${ }^{1}$, XIAOFANG SUN $^{1}$ and WEIHUA WANG ${ }^{1,2}$

\begin{abstract}
${ }^{1}$ Key Laboratory for Major Obstetrics Diseases of Guangdong Province, Key Laboratory of Reproduction and Genetics of Guangdong Higher Education Institutes, The Third Hospital Affiliated of Guangzhou Medical University, Guangzhou, Guangdong 510150, P.R. China; ${ }^{2}$ New Houston Health, Houston, TX 77063, USA
\end{abstract}

Received February 5, 2018; Accepted May 10, 2018

DOI: $10.3892 / \mathrm{mmr} .2018 .9167$

\begin{abstract}
The aim of the present study was to verify the effects of heavy metal coupling agents (sodium citrate and EDTA) and antioxidants (acetyl carnitine and lipoic acid) on the number of oocytes, as well as the ageing of mitochondria, chromosomes and spindles in mice. C57BL/6 female mice were randomly classified into four groups ( $n=12$ per group): i) Heavy metal coupling agent; ii) antioxidant; iii) mixed group; and iv) the normal control group. For the treatments, heavy metal coupling agents and antioxidants were added to the drinking water provided to the mice. Following 3, 6, 9 and 12 months of treatment, the number of oocytes and mitochondrial membrane potential were determined, and chromosome and spindle structures were observed. With increasing age, the experimental mice in the four groups showed significantly decreased numbers of oocytes, reduced mitochondrial activity, and increased rates of spindle and chromosome abnormalities, which indicated age-induced ageing of mouse oocytes; thus, a mouse ageing oocyte model had been successfully established. For mice of the same age, more oocytes, higher mitochondrial activity, and lower spindle and chromosome malformation rates were detected in the antioxidant and mixed groups when compared with the normal control groups. Furthermore, no significant difference in the number of oocytes, mitochondrial activity or chromosome malformation rates was observed between the heavy metal coupling agent group and normal control group, which was possibly due to less metal being absorbed during the breeding process. Therefore, the results
\end{abstract}

Correspondence to: Dr Xiaofang Sun, Key Laboratory for Major Obstetrics Diseases of Guangdong Province, Key Laboratory of Reproduction and Genetics of Guangdong Higher Education Institutes, The Third Hospital Affiliated of Guangzhou Medical University, 63 Duobao Road, Guangzhou, Guangdong 510150, P.R. China

E-mail: xiaofangsun@gzhmu.edu.cn

Dr Weihua Wang, New Houston Health, 2500 Fondren Road, Suite 350, Houston, TX 77063, USA

E-mail: wangweihua11@yahoo.com

Key words: aging, heavy metal coupling agent, antioxidants, oxidative stress demonstrated that the antioxidants acetyl carnitine and lipoic acid may serve a role in delaying oocyte ageing.

\section{Introduction}

Recently, infertility has become one of the more common diseases endangering human health, as a result of the long-term impact of industrialization, and contaminated environments, water and food on human health. In addition to reproductive system diseases, unknown harmful substances are the leading cause of infertility, habitual abortion and embryonic defects $(1,2)$. In China, the number of patients with infertility has increased rapidly in the past 10 years, and infertility currently affects $\sim 15 \%$ of females of reproductive age (3). With the development of detection techniques, such as polymerase chain reaction (PCR), fluorescence in situ hybridization (FISH), and microarray analysis, researchers have demonstrated that the incidence of chromosomal abnormalities in human embryos is very high for women that use assisted reproductive techniques (4). It is considered that the incidence of chromosomal abnormalities in embryos increases with advancing age in females $(5,6)$; however, a significant upward trend in such abnormalities has been observed among young patients (4). Aneuploid embryos are primarily derived from oocytes, and their formation is prevalent among young women and may be associated with various environmental changes.

Extensive studies have confirmed that over $50 \%$ of reproductive and developmental defects are caused by chemical substances and environmental factors, including heavy metals, such as lead, cadmium, nickel and chromium, and organic compounds, including dioxins, aldehydes and phenols (7-9). However, the causes of chromosome abnormalities, as well as the signaling pathways and molecular mechanisms involved in oocytes, remain unclear. It has been reported that heavy metals can trigger oxidative stress, which may reduce the level of antioxidants in cells and also cause abnormal chromosomal division. This finding suggests that the formation of aneuploidy may be associated with the levels of active oxygen and antioxidants in oocytes and that heavy metal coupling agents and antioxidants may effectively reduce chromosomal abnormalities. In the present study, we explored the effects of heavy metal coupling agents and antioxidants on the ageing of mouse oocytes. 


\section{Materials and methods}

Grouping and treatment of mice. Female C57BL/6 mice (body weight, $15 \pm 2 \mathrm{~g}$ ) at 3 weeks of age were obtained from Guangdong Medical Laboratory Animal Center. All experimental animals were housed individually under a $12 / 12 \mathrm{~h}$ light/dark cycle in a specific pathogen-free animal facility at the Laboratory Animal Center of Southern Medical University (Guangdong, China), with controlled temperature $\left(23 \pm 2^{\circ} \mathrm{C}\right)$ and relative humidity (40-60\%). Food and water were available ad libitum during the experimental period. The experimental procedures and animal conditions were approved by the Animal Ethics Committee of The Third Affiliated Hospital of Guangzhou Medical University.

The mice were divided into four groups as described below ( $n=12$ per group). Mice in the heavy metal coupling agent group (group A) were given pure water containing $0.3 \mathrm{mmol} / 1 \mathrm{EDTA}$ and $0.15 \mathrm{mmol} / 1$ sodium citrate (Sigma-Aldrich; Merck KGaA, Darmstadt Germany). Mice in the antioxidant group (group B) were given pure water supplemented with $40 \mathrm{mg} / \mathrm{l}$ lipoic acid and $100 \mathrm{mg} / \mathrm{l}$ acetyl carnitine (GNC Company, Propinsi Lampung, Indonesia). All concentrations were selected on basis of preliminary experiments (data not shown). Mice in the mixed group (group C) were given pure water supplemented with heavy metal coupling agents and antioxidants. Mice in the normal control group (group D) were given pure water. The pure water was processed using aseptic filtration. Mice were randomly selected from each group for subsequent experiments following 3, 6,9 and 12 months of treatment.

Oocyte collection. Female mice were superovulated by intraperitoneal injection of $10 \mathrm{IU}$ of pregnant mare serum gonadotropin (PMSG), followed 46-48 h later by $10 \mathrm{IU}$ of human chorionic gonadotropin (HCG; both Ningbo Second Hormone Factory, Zhejiang, China). Drinking water was provided to mice from each group during the promotion of ovulation. The mice were sacrificed by cervical dislocation after $12-13 \mathrm{~h}$ to collect the oocytes. Briefly, the abdominal cavity was opened exposing the uterus, fallopian tubes and ovaries, followed by separation of blood vessels and adipose tissue. Then, the fallopian tubes were removed and quickly placed in human fallopian tube fluid (HTF) which was artificially prepared, and the fallopian tubes of mice in the same group were grouped together. Following rinsing with HTF three times, the magnum of the fallopian tubes was removed to release cumulus-oocyte complexes (COCs). The COCs were washed twice with HTF and incubated with hyaluronidase (1:9; Invitrogen; Thermo Fisher Scientific, Inc., Waltham, MA, USA) to remove granular lymphocytes and obtain MII-stage nude oocytes. The oocytes were counted following three washes with $\mathrm{HTF}$ and incubated at $37^{\circ} \mathrm{C}$ in a humidified $5 \%$ $\mathrm{CO}_{2}$ incubator in $40 \mu \mathrm{l}$ drops of HTF medium covered with mineral oil (Sigma-Aldrich; Merck KGaA). The mice reared for 12 months did not have a sufficient number of oocytes, thus, only the number of oocytes in this age group was counted.

Evaluation of the mitochondrial membrane potential in mature oocytes using JC-1 dye. A JC-1 mitochondrial membrane potential detection kit (Sigma-Aldrich; Merck KGaA) was used to evaluate the mitochondrial membrane potential. First,
$1 \mathrm{X}$ working solution was prepared with $200 \mathrm{X}$ JC-1, dyeing buffer and pure water according to the manufacturer's protocol, then preheated at $37^{\circ} \mathrm{C}$ in a water bath. Next, $50 \mu \mathrm{l}$ of $1 \mathrm{X}$ working solution was divided into several droplets, which were covered with mineral oil and preheated at $37^{\circ} \mathrm{C}$. Subsequently, 15-20 MII-stage oocytes were incubated for 20-25 min with the working solution at $37^{\circ} \mathrm{C}$ in $5 \% \mathrm{CO}_{2}$, followed by 2-3 washes. JC-1 mitochondrial fluorescence was visualized with a laser-scanning confocal microscope (Nikon-C2Si; Nikon Corp., Tokyo, Japan), and ImageJ software was used for quantitative analysis.

Chromosome examination in mature oocytes. MII-stage oocytes were digested in 3\% trypsin for 2-5 min to clear the zona pellucida and then quickly transferred to the fresh HTF droplets. Appropriately sized circles were drawn on a clean glass slide using a crayon, and each circle was filled with an appropriate amount of 1\% paraformaldehyde (Sigma-Aldrich; Merck KGaA) with 5-10 oocytes. After the nuclear membrane was ruptured, the oocytes were fixed, dried and marked, and stored in a $-20^{\circ} \mathrm{C}$ freezer. For imaging, the glass slides were removed, stained for $10 \mathrm{~min}$ with $10 \mu \mathrm{g} / \mathrm{ml}$ propidium iodide (PI) in the dark, covered with a cover glass, mounted with nail polish, and then stored at $-20^{\circ} \mathrm{C}$. The number of chromosomes was calculated in each oocyte.

Examination of spindle morphology in mature oocytes. First, oocytes were fixed. Briefly, 25 MII-stage oocytes from each group with normal morphology were fixed with $4 \%$ paraformaldehyde at room temperature for $30 \mathrm{~min}$. Following permeabilization with $0.5 \%$ Triton X-100 in phosphate-buffered saline (PBS) for $20 \mathrm{~min}$, the oocytes were blocked in a blocking solution (PBS containing 1\% bovine serum albumin) for $1 \mathrm{~h}$ and then incubated with an $\alpha$-tubulin antibody (Sigma-Aldrich; Merck KGaA) diluted in blocking solution for $1.5 \mathrm{~h}$ at room temperature or overnight at $4^{\circ} \mathrm{C}$. After the oocytes were washed with a washing solution (PBS with $0.1 \%$ Tween-20 and $0.01 \%$ Triton X-100) three times (5 min/wash), the chromosomes were stained with PI (Sigma-Aldrich; Merck KGaA) for $15 \mathrm{~min}$, and the oocytes were then mounted on glass slides and observed using a laser-scanning confocal microscope.

Next, the spindles were observed. In normal oocytes, the spindles exhibit a fusiform shape and have a granular structure at the two poles, with regularly arranged spindle microtubules (green) connecting the spindle poles to the chromosomes (red) that are arranged in order on the equatorial plate. However, in an abnormal state, the spindle length is contracted, the microtubules are irregular or completely absent, and the chromosomes are arranged outside of the equatorial plate, with a dispersed distribution, or only lightly stained.

Statistical analysis. Statistical analyses were performed using SPSS version 13.0 (SPSS Inc., Chicago, IL, USA). All experimental data are presented as percentages and were analyzed using the $\chi^{2}$ test. A repeated-measures analysis of variance was also performed and post hoc multiple factor analysis of variance with Scheffé's test were applied to compare the quantitative data, with $\alpha=0.05$ as a test standard. $\mathrm{P}<0.05$ was considered to indicate a statistically significant difference. 


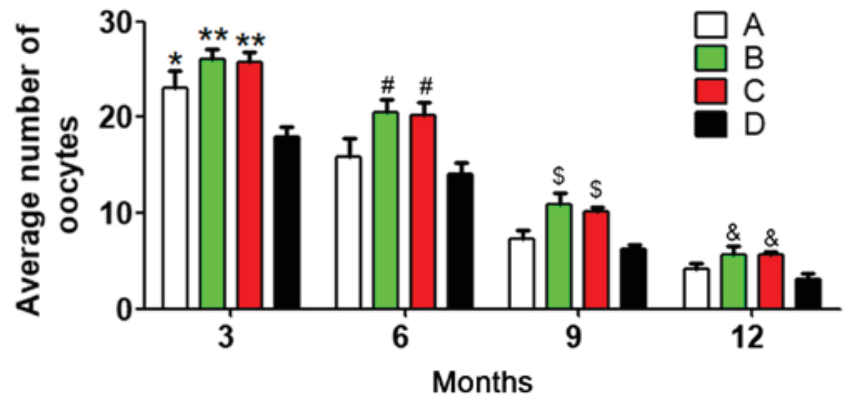

Figure 1. Average number of oocytes in each group at the four time points. A, heavy metal coupling agent group; B, antioxidant group; C, mixed group; and $\mathrm{D}$, normal control group. ${ }^{*} \mathrm{P}<0.05$ and ${ }^{* *} \mathrm{P}<0.01$ vs. group $\mathrm{D}$ at 3 months; ${ }^{\prime} \mathrm{P}<0.05$ vs. group D at 6 months; ${ }^{\$} \mathrm{P}<0.05$ vs. group $\mathrm{D}$ at 9 months; ${ }^{\&} \mathrm{P}<0.05$ vs. group $\mathrm{D}$ at 12 months.

\section{Results}

Antioxidants effectively maintain oocyte numbers. To reduce individual differences, 10 mice were randomly selected from each group at each age stage, and the average number of oocytes was calculated. Analysis of variance and multiple-factor repetitive analysis revealed that the primary effect of age in each group was significantly different $(\mathrm{P}<0.05)$, the differences in the oocyte numbers at different ages were statistically significant $(\mathrm{P}<0.05)$, and different associations were observed between different groups and different age stages $(\mathrm{P}<0.05)$. There were significant differences in the number of oocytes between group $\mathrm{D}$ and the other three groups at 3 months $(\mathrm{P}<0.05)$. In addition, at 6,9 and 12 months, the oocyte number was significantly different between group B and group D, and between group $\mathrm{C}$ and group $\mathrm{D}$ (both $\mathrm{P}<0.05$ ) (Fig. 1). These results demonstrated that with prolonged treatment times, the oocyte number was better-maintained in the antioxidant group compared with that in the heavy metal coupling agent and normal control groups. Meanwhile, the oocyte number in the heavy metal coupling agent group was significantly different from that in the normal control group at 3 months, indicating that heavy metal coupling agents serve a role in maintaining oocyte numbers, but the effect was not as evident as that of antioxidants. One possible reason might be that the mice were not exposed to many heavy metals during treatment, which made the heavy metal coupling agents less useful.

Antioxidants effectivelyalleviate the decrease in mitochondrial activity. In our experiment, mitochondrial activity in mouse oocytes decreased with age (Fig. 2A). As shown in Fig. 2B, in the four groups, mitochondrial activity, represented as red fluorescence, was significantly lower at 9 months compared with at 3 or 6 months. In addition, groups B and C had significantly higher mitochondrial activity levels compared with groups A and B at 9 months. Furthermore, no significant differences were observed in mitochondrial activity between the four groups at 3 and 6 months. These results suggested that antioxidants effectively alleviated the reduction in mitochondrial activity.

Antioxidants reduce the incidence of chromosomal abnormality. As shown in Fig. 3, the incidence of chromosomal abnormality increased with age and/or treatment time in each group. At 3 months, there were no significant differences in chromosome abnormalities between the four groups ( $\mathrm{P}>0.05)$. Interestingly, groups A and D exhibited significantly increased chromosomal abnormalities compared with groups B and C at 6 and 9 months. These results indicated that antioxidants reduced the incidence of chromosomal abnormalities.

Antioxidants reduce the incidence of spindle abnormality. Immunofluorescence analysis was performed to detect the condition of spindles in oocytes (Fig. 4A). As shown in Fig. 4B, the incidence of spindle abnormality increased with age and/or treatment time in each group. Groups $\mathrm{B}$ and $\mathrm{C}$ exhibited no significant differences in spindle abnormality rate at any time point $(\mathrm{P}>0.05)$. There were statistically significant differences in spindle abnormalities between group A and group $\mathrm{D}$ at the same time points $(\mathrm{P}<0.05)$. At 6 and 9 months, the spindle abnormalities in groups $\mathrm{B}$ and $\mathrm{C}$ were remarkably lower compared with that in groups A and D. These results suggested that antioxidants reduced the incidence of spindle abnormalities.

\section{Discussion}

Oocyte ageing, and germ cell dysfunction and depletion, accompanied by follicular and ovarian atrophy and endocrine abnormalities, have been widely suggested to be the major causes of reproductive ageing, menopause and associated pathologies, including infertility $(10,11)$. It has been reported that oxidative stress serves an important role in oocyte ageing, and increased levels of reactive oxygen species (ROS) have been identified in the follicular fluid of elderly women $(12,13)$. Oxidative stress is defined as an imbalance between increased levels of ROS and low activity of antioxidative mechanisms. A variety of destructive stimuli can cause the overproduction of highly reactive molecules, such as ROS and reactive nitrogen species (RNS), whereby the antioxidative mechanisms are unable to eliminate them. A high concentration of ROS can destroy DNA, protein and lipids, leading to impairments in gene expression, damage to cell structures and functions, and even cell death, which is considered to be a major mechanism involved in organism ageing and diseases. Previous studies have demonstrated that a high level of ROS is associated with reduced meiosis $(14,15)$, chromosome abnormalities, spindle microtubule deformation (16-19) and reduced embryonic development potential $(20,21)$. These findings suggest that oxidative stress may partially contribute to the decline in fertility that is directly associated with oocyte ageing. Thus, we speculated that oocyte ageing might be effectively blocked by reducing ROS via antioxidant supplementation.

There are two types of antioxidant defense systems in the human body: The enzymatic antioxidant system, including superoxide dismutase (SOD), catalase (CAT) and glutathione peroxidase (GSH-Px); and the non-enzymatic antioxidant system, including glutathione, $\alpha$-lipoic acid, carotenoids, and vitamin C. Our study revealed that the number and quality of mouse oocytes increased following prolonged treatment with the antioxidants lipoic acid and acetyl carnitine. Consistent with these results, Tarín et al (22) reported that oral administration of vitamin $\mathrm{C}$ and vitamin $\mathrm{E}$ could also offset the negative 


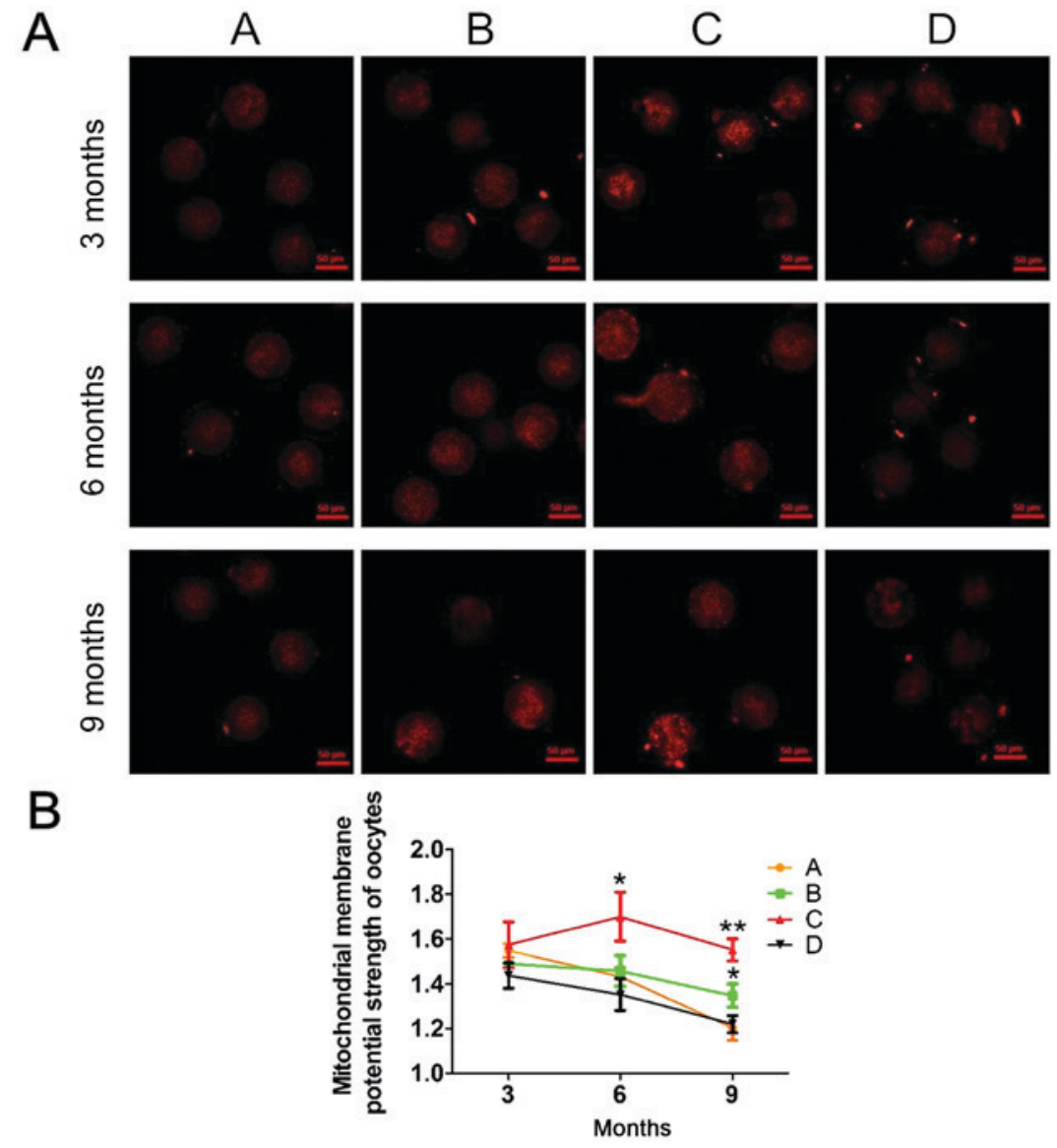

Figure 2. Fluorescent images of mitochondrial activity in each group at 3,6 and 9 months. (A) The red fluorescence was evidently weaker in oocytes from older mice when compared with that observed in those from younger mice. Scale bars, $50 \mu \mathrm{m}$. (B) The trends in the mitochondrial membrane potential strength of oocytes in each group. A, heavy metal coupling agent group; B, antioxidant group; C, mixed group; and D, normal control group. Following JC-1 staining, red fluorescence in the TRITC channel indicated mitochondrial activity when the membrane potential was activated. Mitochondrial membrane potential strength=mitochondrial activity/apoptosis (the ratio of red to green); the greater the membrane potential strength, the greater the activity. ${ }^{*} \mathrm{P}<0.05$ and ${ }^{* *} \mathrm{P}<0.01$ vs. group $\mathrm{D}$.

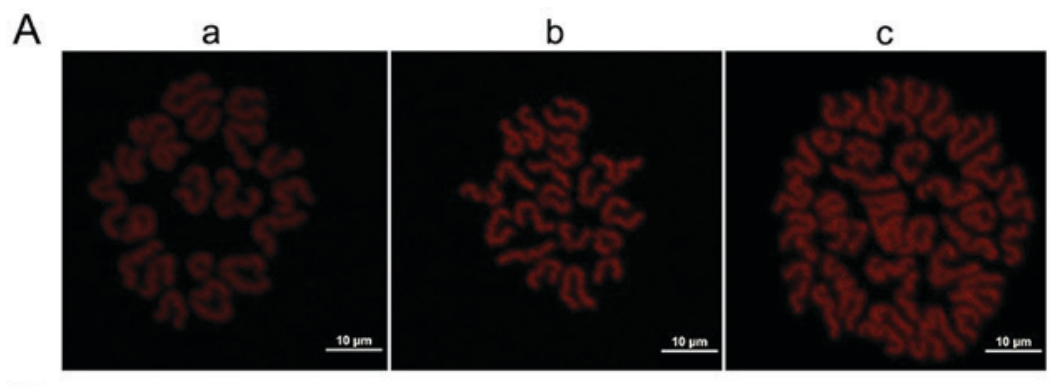

B

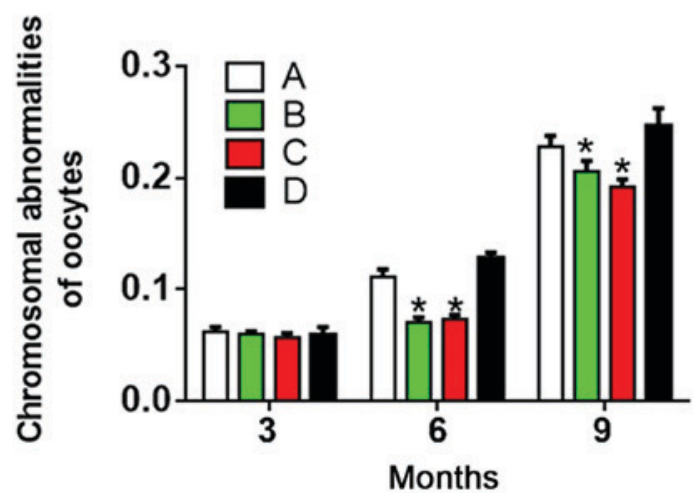

Figure 3. Fluorescent images of chromosome structure in oocytes. (A) Fluorescent images of chromosome structure in oocytes: Image a, normal chromosomes $(\mathrm{n}=20)$; $\mathrm{b}$, abnormal chromosomes $(\mathrm{n}=19)$; and $\mathrm{c}$, abnormal chromosomes $(\mathrm{n}=40)$. Scale bars, $10 \mu \mathrm{m}$. (B) Chromosomal abnormalities in oocytes in each group. $\mathrm{A}$, heavy metal coupling agent group; B, antioxidant group; $\mathrm{C}$, mixed group; and $\mathrm{D}$, normal control group. " $\mathrm{P}<0.05$ vs. group $\mathrm{D}$. 


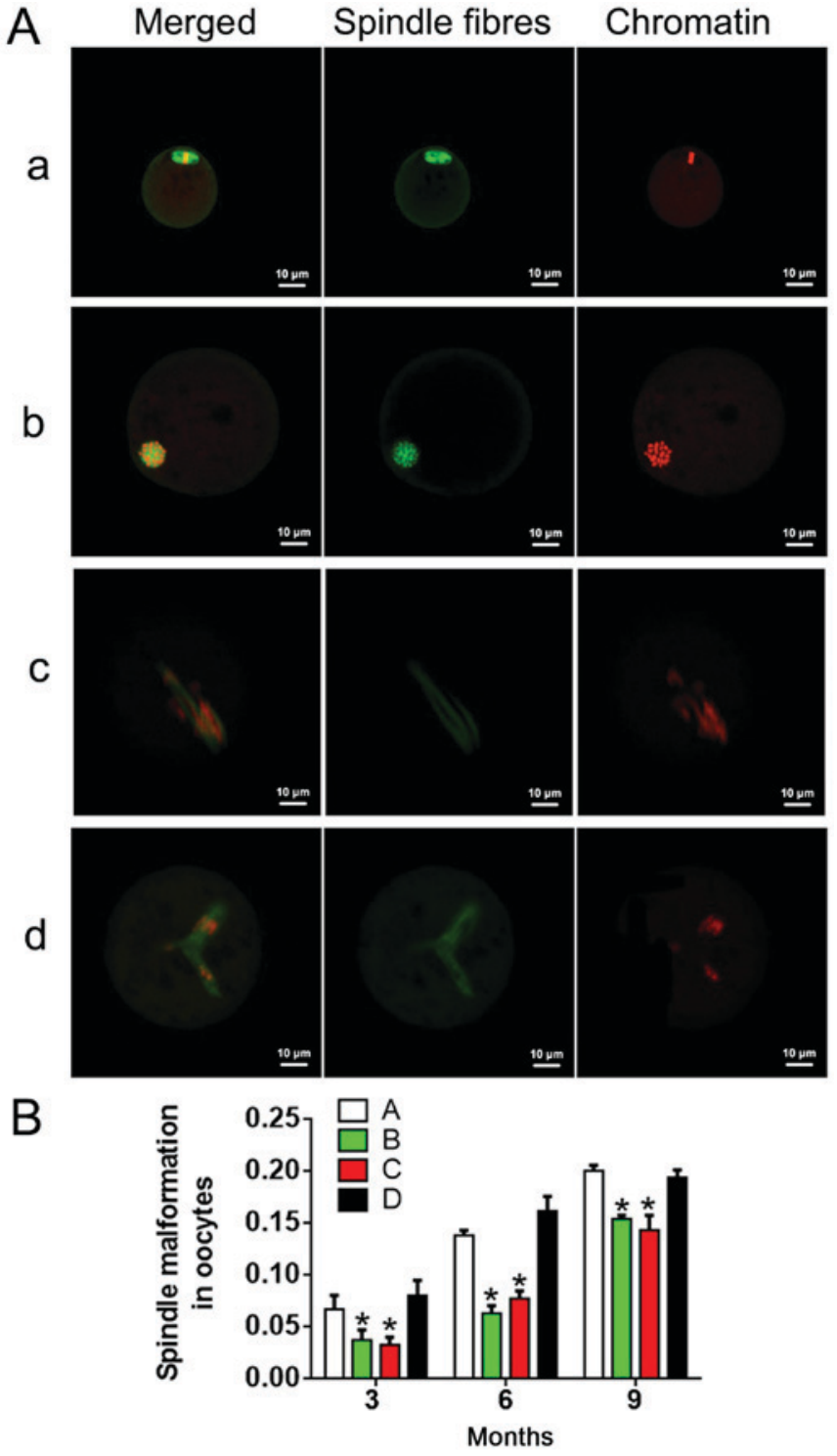

Figure 4. Fluorescent images of chromosomes and spindle structures in oocytes. (A) Fluorescent images of chromosomes and spindle structures in oocytes: Images a and b present normal spindle and chromosomes; cross-sections are shown in image a, and longitudinal sections are shown in image b. Images $\mathrm{c}$ and d present abnormal spindle and chromosomes. Green indicates spindle fibers, and red indicates chromatin. Scale bars, $10 \mu \mathrm{m}$ (B) Spindle malformation in oocytes in each group. A, heavy metal coupling agent group; B, antioxidant group; C, mixed group; and D, normal control group. ${ }^{*} \mathrm{P}<0.05$ vs. group $\mathrm{D}$.

effects of female ageing on the number and quality of oocytes, and the response to oxidative stress has clinical significance in preventing female age-associated aneuploidy (23). Moreover, Liu et al (24) demonstrated that the quality and number of oocytes significantly increased following $\mathrm{N}$-acetyl-L-cysteine treatment. Collectively, these studies suggest that antioxidants exert positive effects on oocytes.

Increased aneuploidy is considered an important indicator of oocyte senescence. In 1965, Harman first proposed the Mitochondrial Free Radical Theory of Aging, postulating that the destruction of DNA and cell membranes caused by peroxidation is the primary reason for the decline in cell function with age (25). Soon afterward, in 1972, Harman put forward the concept of mitochondrial senescence (26). Mitochondria are the most abundant organelles in oocytes, providing energy for various chemical reactions and other organelles. Thus, with fewer mitochondria, the chemical reactions in oocytes cannot easily proceed, reducing the activity of oocytes and embryos, and subsequently leading to decreased fertility and increased aneuploidy. Recently, an increasing number of studies have focused on the association between mitochondrial metabolism and oocyte maturation, fertilization and early embryonic development $(15,27,28)$. It has been reported that oocyte aging, mitochondrial apoptosis and a decrease in mitochondrial membrane potential are associated with increased age (29). In our study, mitochondrial activity in oocytes was significantly lower at 9 months compared with at 3 and 6 months, which supports the findings by Tarín et al (12), whereby there is a significant correlation between age and oxidative stress in mammals (15). Additionally, mitochondrial activity was significantly decreased in the antioxidant group compared with the heavy metal coupling agent and normal control groups, indicating that antioxidants could effectively maintain the activity of mitochondria, thus, deferring the process of oocyte senescence. It is generally known that spindles serve a regulatory role in chromosome arrangement and separation $(30,31)$. The majority of aneuploid embryos originate from the two meiotic divisions, particularly from the initial meiosis $(32,33)$. In this study, we examined the structures of chromosomes and spindles, and found that chromosomal and spindle abnormalities increased in an age-dependent manner. We also confirmed that antioxidants could effectively remove excess ROS in cells and maintain mitochondrial activity, thereby protecting the normal morphology of spindles and chromosomes in oocytes.

It is traditionally considered that the number of female oocytes is fixed at birth and decreases at various rates during life until the ovarian reserve is exhausted, and menopause is reached (34). The results of this study confirmed that ovarian senescence indicated a decline in the number and quality of oocytes. In addition, our experiments demonstrated that lipoic acid and acetyl carnitine could not prevent the negative effects caused by ovarian ageing but could significantly delay this process. However, heavy metal coupling agents, including EDTA and sodium citrate, showed no significant effect on oocytes, possibly because the experimental mice were not exposed to heavy metals. Understandably, research on the mechanism of oocyte ageing is of great significance for women seeking to use assisted reproductive strategies.

In conclusion, our data demonstrated that lipoic acid and acetyl carnitine can delay the ageing process of germ cells, which may be achieved by protecting oocytes against senescence via the maintenance of mitochondrial activity, and prevention of chromosomal and spindle abnormalities.

\section{Acknowledgements}

The authors would like to thank the Laboratory Animal Center of Guangzhou Medical University (Guangdong, China) for providing the animal breeding sites.

\section{Funding}

The present study was supported by the National Natural Science Foundation of China (grant no. 81270745), the Science 
and Information Technology of Guangzhou Key Project (grant no. 201508020258) and Guangdong Province Science and Technology Project (grant no. 2016B030229008).

\section{Availability of data and materials}

All data generated or analyzed during this study are included in this published article.

\section{Authors' contributions}

YXX, XFS and WHW conceived and designed the study. YXX, LFL, STQ, BS, SMO and YHX performed the experiments. YXX and YJX conducted the statistical analysis. YXX, XFS and WHW wrote the paper. All authors read and approved the final manuscript.

\section{Ethics approval and consent to participate}

The experimental procedures and animal conditions were approved by the Animal Ethics Committee of the Third Affiliated Hospital of Guangzhou Medical University (Guangzhou, China).

\section{Patient consent for publication}

Not applicable.

\section{Competing interests}

The authors declare that they have no competing interests.

\section{References}

1. Herrera LA, Prada D, Andonegui MA and Dueñas-González A The epigenetic origin of aneuploidy. Curr Genomics 9: 43-50, 2008.

2. Dulskiene V and Maroziene L: Impact on environmental factors on the reproductive system and fetal development. Medicina (Kaunas) 38: 1072-1077, 2002 (In Lithuanian).

3. Montoya JM, Bernal A and Borrero C: Diagnostics in assisted human reproduction. Reprod Biomed Online 5: 198-210, 2002.

4. Baart EB, Martini E, van den Berg I, Macklon NS, Galjaard RJ, Fauser BC and Van Opstal D: Preimplantation genetic screening reveals a high incidence of aneuploidy and mosaicism in embryos from young women undergoing IVF. Hum Reprod 21: 223-233, 2006.

5. Jones KT: Meiosis in oocytes: Predisposition to aneuploidy and its increased incidence with age. Hum Reprod Update 14 $143-158,2008$

6. Chiang T, Schultz RM and Lampson MA: Meiotic origins of maternal age-related aneuploidy. Biol Reprod 86: 1-7, 2012.

7. Zenzes MT: Smoking and reproduction: Gene damage to human gametes and embryos. Hum Reprod Update 6: 122-131, 2000.

8. Kumar S: Occupational exposure associated with reproductive dysfunction. J Occup Health 46: 1-19, 2004.

9. Gerhard I, Waibel S, Daniel V and Runnebaum B: Impact of heavy metals on hormonal and immunological factors in women with repeated miscarriages. Hum Reprod Update 4: 301-309, 1998.

10. Tatone C, Amicarelli F, Carbone MC, Monteleone P, Caserta D, Marci R, Artini PG, Piomboni P and Focarelli R: Cellular and molecular aspects of ovarian follicle ageing. Hum Reprod Update 14: 131-142, 2008.

11. Coccia ME and Rizzello F: Ovarian reserve. Ann N Y Acad Sci 1127: 27-30, 2008
12. Tarín JJ: Potential effects of age-associated oxidative stress on mammalian oocytes/embryos. Mol Hum Reprod 2: 717-724, 1996.

13. Tarín JJ: Aetiology of age-associated aneuploidy: A mechanism based on the 'free radical theory of ageing'. Hum Reprod 10: $1563-1565,1995$.

14. TamuraH,Takasaki A,MiwaI,TaniguchiK, MaekawaR,AsadaH, Taketani T, Matsuoka A, Yamagata Y, Shimamura K, et al: Oxidative stress impairs oocyte quality and melatonin protects oocytes from free radical damage and improves fertilization rate. J Pineal Res 44: 280-287, 2008.

15. Chaube SK, Prasad PV, Thakur SC and Shrivastav TG: Hydrogen peroxide modulates meiotic cell cycle and induces morphological features characteristic of apoptosis in rat oocytes cultured in vitro. Apoptosis 10: 863-874, 2005.

16. Zhang X, Wu XQ, Lu S, Guo YL and Ma X: Deficit of mitochondria-derived ATP during oxidative stress impairs mouse MII oocyte spindles. Cell Res 16: 841-850, 2006.

17. Tarín JJ, Vendrell FJ, Ten J, Blanes R, van Blerkom J and Cano A: The oxidizing agent tertiary butyl hydroperoxide induces disturbances in spindle organization, c-meiosis, and aneuploidy in mouse oocytes. Mol Hum Reprod 2: 895-901, 1996.

18. Liu L and Keefe DL: Ageing-associated aberration in meiosis of oocytes from senescence-accelerated mice. Hum Reprod 17: 2678-2685, 2002.

19. Choi WJ, Banerjee J, Falcone T, Bena J, Agarwal A and Sharma RK: Oxidative stress and tumor necrosis factor-alpha-induced alterations in metaphase II mouse oocyte spindle structure. Fertil Steril 88 (4 Suppl): S1220-S1231, 2007.

20. Tarín JJ, Gómez-Piquer V, Pertusa JF, Hermenegildo C and Cano A: Association of female aging with decreased parthenogenetic activation, raised MPF, and MAPKs activities and reduced levels of glutathione S-transferases activity and thiols in mouse oocytes. Mol Reprod Dev 69: 402-410, 2004.

21. Bhattacharya S, Maheshwari A and Mollison J: Factors associated with failed treatment: An analysis of 121,744 women embarking on their first IVF cycles. PLoS One 8: e82249, 2013.

22. Tarín JJ, Pérez-Albalá S and Cano A: Oral antioxidants counteract the negative effects of female aging on oocyte quantity and quality in the mouse. Mol Reprod Dev 61: 385-397, 2002.

23. Tarín JJ, Vendrell FJ, Ten J and Cano A: Antioxidant therapy counteracts the disturbing effects of diamide and maternal ageing on meiotic division and chromosomal segregation in mouse oocytes. Mol Hum Reprod 4: 281-288, 1998.

24. Liu J, Liu M, Ye X, Liu K, Huang J, Wang L, Ji G, Liu N, Tang X, Baltz JM, et al: Delay in oocyte aging in mice by the antioxidant N-acetyl-L-cysteine (NAC). Hum Reprod 27: 1411-1420, 2012.

25. Harman D: The free radical theory of aging: Effect of age on serum copper levels. J Gerontol 20: 151-153, 1965.

26. Harman D: The biologic clock: The mitochondria? J Am Geriatr Soc 20: 145-147, 1972.

27. Takahashi T, Takahashi E, Igarashi H, Tezuka N and Kurachi H: Impact of oxidative stress in aged mouse oocytes on calcium oscillations at fertilization. Mol Reprod Dev 66: 143-152, 2003.

28. Liu L, Trimarchi JR and Keefe DL: Involvement of mitochondria in oxidative stress-induced cell death in mouse zygotes. Biol Reprod 62: 1745-1753, 2000.

29. Zhang D, Keilty D, Zhang ZF and Chian RC: Mitochondria in oocyte aging: Current understanding. Facts Views Vis Obgyn 9: 29-38, 2017.

30. Wang WH and Sun QY: Meiotic spindle, spindle checkpoint and embryonic aneuploidy. Front Biosci 11: 620-636, 2006.

31. Vogt E, Kirsch-Volders M, Parry J and Eichenlaub-Ritter U: Spindle formation, chromosome segregation and the spindle checkpoint in mammalian oocytes and susceptibility to meiotic error. Mutat Res 651: 14-29, 2008

32. Hassold T and Hunt P: To err (meiotically) is human: The genesis of human aneuploidy. Nat Rev Genet 2: 280-291, 2001.

33. Hassold T, Hall $\mathrm{H}$ and Hunt P: The origin of human aneuploidy: Where we have been, where we are going. Hum Mol Genet 16 Spec No. 2: R203-R208, 2007.

34. Djahanbakhch O, Ezzati M and Zosmer A: Reproductive ageing in women. J Pathol 211: 219-231, 2007.

This work is licensed under a Creative Commons Attribution-NonCommercial-NoDerivatives 4.0 International (CC BY-NC-ND 4.0) License. 